\title{
NEGATION AS CANCELLATION, CONNEXIVE LOGIC, AND qLPm
}

\author{
HEINRICH WANSING AND DANIEL SKURT
}

\begin{abstract}
In this paper, we shall consider the so-called cancellation view of negation and the inferential role of contradictions. We will discuss some of the problematic aspects of negation as cancellation, such as its original presentation by Richard and Valery Routley and its role in motivating connexive logic. Furthermore, we will show that the idea of inferential ineffectiveness of contradictions can be conceptually separated from the cancellation model of negation by developing a system we call $q L P m$, a combination of Graham Priest's minimally inconsistent Logic of Paradox with q-entailment (quasi-entailment) as introduced by Grzegorz Malinowski.
\end{abstract}

Keywords Negation as cancellation, Connexive logic, Conjunctive simplification, Minimally inconsistent $L P$, q-entailment, Non-monotonic logic, Paraconsistent logic

\section{INTRODUCTION}

In the present paper, we shall consider three interrelated issues: the so-called cancellation view of negation, its relationship to connexive logic (both as presented by Routley and Routley [27] and Routley $[25,26])$, and the inferential role of contradictions. In particular, we shall make the following points:

- Since the cancellation view of negation is problematic, it is better not seen as a conceptual basis of anything, and should therefore most definitely not be used as a conceptual basis of connexive logic.

- The rejection of the cancellation view of negation has repercussions on the conception of connexive logic. If negation as cancellation is used to motivate Aristotle's and Boethius' theses as characteristic principles of connexive logic, the failure of Conjunctive Addition (and the failure of Disjunctive Simplification, if the latter is equivalent in the connexive logic under consideration) may be regarded as a kind of collateral damage. Giving up the cancellation view of negation enables a different perspective on connexive logic.

- Although Routley [26] ${ }^{1}$ and Routley and Routley [27] introduce negation as cancellation (erasure, etc.) as assigning no inferential role to contradictions (or at most a very limited one), the idea of inferential ineffectiveness of contradictions can be conceptually separated from the cancellation model of negation. The proper context of inferentially ineffective contradictions is non-monotonic reasoning.

- This perspective is exemplified by realizing that the paraconsistent negation in Graham Priest's minimally inconsistent Logic of Paradox [22], in combination with a certain non-standard, but well understood notion of entailment, namely q-entailment (quasi-entailment) [14], guarantees $e x$ contradictione non sequitur contradictio ("from a contradiction no contradiction follows").

The latter observation is not meant to endorse the presented system, $q L P m$, as a non-monotonic logic that is superior to other related systems, but is merely a formal observation. In particular, we do not intend

\footnotetext{
${ }^{1}$ Relevance Logics and Their Rivals. Vol. 1 is authored by "Richard Routley with Robert K. Meyer, Val Plumwood and Ross T. Brady", but "[t]he text has been almost entirely written by the first author" [26, $x v]$. We here ascribe the discussion of negation as cancellation in [26] to Richard Routley.

Australasian Journal of Logic (15:2) 2018, Article no. 3.11
} 
to advocate or promote q-entailment as the ultimate basis for obtaining a system in which contradictions are inferentially ineffective. ${ }^{2}$

In Section 2, we present a critical discussion of negation as cancellation and negation as a propositional analogue of subtraction in mathematics. Section 3 is devoted to the relationship between negation as cancellation and connexive logic. It is well-known that the motivation for connexive logic is diverse, and there is still some discussion about what exactly connexive logic is, cf. [32]. The Routleys explain that the cancellation view of negation leads to connexive logic, and since the cancellation model comes with a commitment to the failure of Conjunctive Simplification $((A \wedge B) \vdash A$ and $(A \wedge B) \vdash B)$, they consider the failure of Conjunctive Simplification as a characteristic feature of connexive logic. Since we reject the cancellation model of negation, we obtain a different view of connexive logic that does not come with a commitment to the failure of Conjunctive Simplification. The issue of inferential ineffectiveness of contradictions is then taken up in Section 4, where we introduce the system $q L P m$ and present some of its properties. The concluding Section 5 contains a brief summary.

\section{NeGATION AS CANCELlation AND NEGATION AS ADDitive INVERSE}

In [27], Richard Routley (later Richard Sylvan) and Valerie Routley (later Val Plumwood) present and discuss various conceptions of negation, depending on the inferential role associated with contradictions. ${ }^{3}$

According to Routley and Routley [27, p. 205]:

Theories of negation differ, very obviously, in the roles they allow or assign to, contradictions. Contradictions may be allowed no inferential role (they imply nothing, except perhaps themselves), a total inferential role (they imply everything), or some limited inferential role (they imply some things, such as their contradictory components, but not others).

The first role assigned to contradictions is usually said to be characteristic of negation as cancellation, the second role of contradictions is familiar from classical and intuitionistic logic, whereas the third role assigned to contradictions is typical of paraconsistent negations in paraconsistent logics such as Priest's Logic of Paradox, LP [21], Belnap and Dunn's first-degree entailment logic, FDE (see [18] for a survey and additional references), or Nelson's constructive four-valued logic $\mathbf{N} 4[1,17,12]$. The Routleys' distinction between theories of negation has been taken up by Graham Priest [23], who refers to the limited-inferential-role account of negation as a partial account of negation. Note that if a logic implements the cancellation view of negation, it will also be paraconsistent because the ex contradictione quodlibet principle, $(A \wedge \sim A) \vdash B$, will not be valid. Moreover, note that the Routleys present the first role assigned to contradictions in two variants, a strict one, according to which a contradiction implies nothing at all, and a moderate one, according to which a contradictory formula $(A \wedge \sim A)$ implies nothing except itself; in particular $(A \wedge \sim A)$ does not imply its conjuncts. The moderate conception concedes very limited inferential role to contradictions and thus, strictly speaking, is a partial account of negation.

The cancellation view of negation is described by Routley and Routley [27, p. 205] as follows:

$\sim A$ deletes, neutralizes, erases, cancels $A$ (and similarly, since the relation is symmetrical, $A$ erases $\sim A$ ), so that $\sim A$ together with $A$ leaves nothing, no content. The conjunction of $A$ and $\sim A$ says nothing, so nothing more specific follows. In particular, $A \wedge \sim A$ does not entail $A$ and does not entail $\sim A$.

\footnotetext{
${ }^{2}$ Independently of Malinowski's work, q-entailment has later been studied by P. Cobreros et al. [5] under the name "TSconsequence" (tolerant strict consequence) in the context of reasoning about vague predicates. More recently, q-entailment has been subsumed under a two-dimensional notion of so-called B-consequence relations, see [3].

${ }^{3}$ Note that the concept of a contradiction is a colorful notion. According to Patrick Grimm [10, p. 55], on a very conservative account, there are "some 240 senses of contradiction". It seems adequate to assume that the Routleys think of contradictions primarily as formulas of the form $A \wedge \sim A$ or $\sim A \wedge A$, or as pairs $\{A, \sim A\}$.
}

Australasian Journal of Logic (15:2) 2018 Article no. 3.11 
This description leaves room for the moderate conception of inferential ineffectivenes of contradictions. The idea is that both $(\sim A \wedge A)$ and $(A \wedge \sim A)$ are void of semantic content, with the effect that nothing more specific follows. ${ }^{4}$ The cancellation view so described, is, however, highly problematic. If we take the deletion, neutralization, erasure, cancellation metaphors literally and seriously, it is not clear in which relation $\sim(\sim A \wedge A)$ and $(\sim A \wedge A)$ stand to each other. How could $\sim(\sim A \wedge A)$ possibly erase $(\sim A \wedge A)$ if $(\sim A \wedge A$ ) leaves nothing at all? How could nothing, $(\sim A \wedge A)$, possibly erase anything? Moreover, what is the outer negation in $\sim(\sim A \wedge A)$ operating upon if it is not operating on semantic content? What is the outer negation in $\sim(\sim A \wedge A)$ operating upon? If the outer negation of a doubly negated formula $\sim A$ is assumed to operate on the inner negation instead of the content of $\sim A$, this would make the meaning of negation as cancellation dependent on syntactic context: in front of an even number of negation signs (or at least in front of a non-negated formula), it would erase semantic content, in front of an uneven number of negation signs, it would withdraw erasure.

In $[26$, p. 89] Routley diagnoses a general problem with negation as cancellation, deletion, or removal. These pictures "confuse statings with statements, the condition stated by $p$ with the performance of asserting, in some mode, $p$," and examples given for negation as cancellation "are all examples of undoing or deleting the performance of asserting $p$, not the undoing or unmaking of the condition $p$ itself."

Routley [26, p. 92] rejects the simple deletion account of negation also for another reason. It would prevent us distinguishing distinct contradictions. As $A \wedge \sim A=B \wedge \sim B$, since they have the same content, namely none, $A \wedge \sim A \rightarrow B \wedge \sim B$, contravening relevance, and yielding paradoxes.

This passage as well as the claim that on the deletion account of negation nothing specific follows from a contradiction raises the question of the relationship between negation as cancellation and the assumed concept of entailment or valid implication. In [25, p. 395], Routley explains that (notation adjusted) "the conjoined content, of $A \wedge \sim A$, is less than that of $A$ and of that of $\sim A$. But implication requires content inclusion, so these (degenerate) examples of Simplification fail." Hence the idea is that the content (meaning) of the consequent of a valid implication must be included in the content (meaning) of the antecedent. As to a meaning or content requirement on valid implications, Routley [25, p. 393] remarks that this "requirement coincides with the broad requirement of relevance: for if antecedent and consequent enjoy a meaning connexion then they are relevant in meaning to one another, and if they are relevant in meaning to one another then they have through the relevance relation a connexion in meaning. Thus the general classes of connexive and relevant logics are one and the same." Whereas relevance logic is normally taken to require that Anderson and Belnap's variable sharing property holds, saying that if an implication $(A \rightarrow B)$ is valid, then $A$ and $B$ share an atomic formula, the stricter relevance criterion of so-called logics of analytic implication requires that if $(A \rightarrow B)$ is valid, then every atomic formula that occurs in $B$ also occurs in $A$. The latter inclusion requirement is known as the conditional version of the "proscriptive principle" (see [9] for an in-depth investigation of logics with analytic implication, as well as references to the relevant literature). Routley argues that the cancellation account of negation validates $A \wedge \sim A \rightarrow B \wedge \sim B$ and thus leads to a violation of relevance. Moreover, the above passage from [26, p. 92] strongly suggests that for Routley the identity of content of formulas $A$ and $B$ guarantees the validity of $A \rightarrow B$, which - in view of violation of relevance - underlines the understanding of content as meaning.

One may consider a more abstract analysis of content in terms of assigning subject matter to formulas, so as to capture what they are about (instead of understanding content as meaning and requiring variable sharing or variable containment for valid implications as a relevance criterion). One such approach has

\footnotetext{
${ }^{4}$ The fact that the content in question is semantic content becomes clear from [25], where content connection is explicitly understood as meaning connection. For present purposes, it not necessary to settle on a specific account of semantic content, or to decide on whether semantic content is characterized in terms of extensions, intensions, hyperintensions, extension/antiextension pairs, situations, structured proposition, or other entities.
}

Australasian Journal of Logic (15:2) 2018 Article no. 3.11 
been developed by Routley himself in a paper on what he called "relevant containment logics" [29]. ${ }^{5}$ In that paper, Sylvan (i.e., Routley) introduces a binary lattice-ordered content containment relation on the set of formulas satisfying the condition that $A$ contains $B$ whenever every atomic formula that occurs in $B$ also occurs in $A$. Sylvan then semantically defines a conditional by assuming the truthconditions of implications in the ternary Routley-Meyer semantics for relevance logics and by demanding, in addition, containment of the consequent in the antecedent of the conditional. ${ }^{6}$ Another option for relating containment and entailment is given by defining containment in terms of a notion of entailment and subject-matter inclusion. Such a proposal can be found in Yablo's [33, p. 3] account of semantic containment:

$A$ contains $B$, I propose, if the argument $A$, therefore $B$, is both truth-preserving and subject-matter preserving.

However, on the more abstract analysis of content, the claim that "the conjoined content, of $A \wedge \sim A$, is less than that of $A$ and of that of $\sim A$ " would not make sense for a categorematic account of content, according to which logical vocabulary does not contribute to content. Note also that although Sylvan [29] does develop a categorematic theory, he remarks that a categorematic account of content containment is "decidedly dubious" [29, p. 15].

We need not pursue this topic any further because, as a result of his own critical considerations on negation as cancellation, Routley [26, p. 89] comes to explaining that negation as deletion is "better viewed as a propositional analogue of the familiar operation of subtraction in mathematics":

Just as the negative integer $-\mathrm{a}$ is obtained by the subtraction operation from positive integer a, so on the subtraction view of negation the negative proposition $\sim A$ is obtained by negation from the positive proposition $A$, and similarly where $B$ is negative (i.e. ultimately of the form $\sim C$ ) the positive proposition $\sim B$ (ultimately $C$ ) is its negation. And just as a positive number added to its negative counterpart gives zero, so a positive proposition $A$ conjoined with its negative $\sim A$ is seen as giving zero $(A$-) content.

In view of the damaging effect of the simple deletion account of negation on relevance, however, Routley [26, p. 92 f.] suggests adjusting the simple subtraction account of negation by generalizing the arithmetic:

The adjustment is by way of the relativisation already hinted at: $\sim A$ deletes $A$ content, and $A$ and $\sim A$ together say nothing $A$-wise, i.e. $A \wedge \sim A$ has no $A$ content. But to say $A \wedge \sim A$ have no $A$ content is not to say it has no $B$ content, so the unfortunate outcome $[\ldots]$ that all contradictions say the same, namely nothing, is avoided [...]. Correspondingly the arithmetical model for connexivism is to be sought not in ordinary arithmetic with its single line through zero, but in generalised arithmetic (as outlined in Kleene 52) with many zeros - one for each distinct statement - and many sequences - one by positive (and negative) extension from each zero.

Since negation, from a semantical point of view, is a unary operation on propositions, we might think of negation as additive inverse (which indeed is sometimes called "negation"), the operation on the set of real numbers that maps a number $a$ to its opposite, the number that, when added to $a$, yields zero. Subtraction is then defined as the addition of the opposite: $a-b=a+(-b)$. A negated formula $\sim A$ would then stand for the opposite of a number, conjunction for addition, and $A \wedge \sim A$ would have zero $A$-content, $0_{A}$. If the content of $B$ is different from the content of $A, B \wedge \sim B$ would have zero $B$-content, and $0_{A} \neq 0_{B}$. Since the additive inverse of 0 is 0 , both $\sim(A \wedge \sim A)$ and $(A \wedge \sim A)$ then have the same zero $A$-content, which is unproblematic for the categorematic conception of content. However, if identity of content guarantees

\footnotetext{
${ }^{5}$ We are grateful to an anonymous reviewer for drawing our attention to this paper.

${ }^{6}$ More recently such a kind of aboutness filter on the truth conditions of conditionals has been imposed in Franz Berto's [2] logic of imagination episodes.
}

Australasian Journal of Logic (15:2) 2018 Article no. 3.11 
mutual entailment, as assumed by Routley in [26], we do have a problem for negation as additive inverse because, in particular, $\sim(A \wedge \sim A)$ would entail $(A \wedge \sim A)$. Moreover, the adjusted arithmetical model of negation has not been worked out in any detail by Routley, and several other problems remain. Negated formulas may occur as disjuncts or as relata of the implication sign, but what are the arithmetical counterparts of disjunction and implication? How would one incorporate quantification?

We may thus summarize that the metaphoric notion of negation as cancellation is conceptually unclear and that its replacement by negation as subtraction in generalized arithmetic is unclear at least insofar as it has not been worked out in detail, and in particular not for a logical vocabulary that contains operations in addition to a unary connective taken to express a kind of negation and a binary connective understood as a kind of conjunction.

\section{Connexive Logic}

Routley and Routley [27, p. 205] continue their characterization of negation as cancellation by saying that "the cancellation (erasure, or neutralization) model leads towards connexivism". However, insofar as the idea of negation as cancellation is unclear, it does not provide a solid conceptual basis of anything, and therefore it does not lead towards connexive logic, or connexivism, as Routley [26] and Routley and Routley [27] call it, in a convincing way. So what is a connexive logic? According to Routley [26, p. 82], connexivism has two leading theses, namely:

1. Simplification $(A \wedge B \rightarrow A, A \wedge B \rightarrow B)$ fails to hold, and its use (and that of its outcome, Addition $)^{7}$ is what is responsible for the paradoxes of implication, not the ancient and correct principles of Disjunctive Syllogism or Antilogism.

2. Every statement is self-consistent, symbolically $A \diamond A$, where the relation of consistency with, symbolised $\diamond$, is interconnected with implication in the standard fashion: $A \diamond B \leftrightarrow$. $\sim(A \rightarrow \sim B)$.

How can connexive logic be detached from the cancellation view of negation? In the first place, the cancellation view is used to arrive at the failure of Simplification. In [27, p. 205] the motivation is described as extending to Aristotle's thesis $\sim(A \rightarrow \sim A)$ : "if $A$ were to entail $\sim A$, it would include as part of it its content, what neutralizes it, $\sim A$, in which event it would entail nothing, having no content. So it is not the case that $A$ entails $\sim A$, that is Aristotle's thesis $\sim(A \rightarrow \sim A)$ holds." The idea is that if a proposition $A$ contains its own neutralizer, $A$ must be void of content, which is impossible. This reasoning is not obvious. If $A$ and $\sim A$ "taken together" leave nothing, why should containment of $\sim A$ in $A$, expressed by $A \rightarrow \sim A$, have the same effect? This would be obvious if the content of the conjunction $A \wedge \sim A$ is identified with the content of the implication $A \rightarrow \sim A$, which is, however, bizarre if content is identified with meaning. In a footnote, Routley and Routley [27, p. 227] remark that the argument assumes that inner and outer negations in Aristotle's thesis are the same, so that the outer negation, which is being paraphrased as "it is not the case that", is negation as cancellation. But what does $\sim(A \rightarrow \sim A)$ erase if $A \rightarrow \sim A$ is not the case?

As to the validity of Boethius' thesis, $(A \rightarrow B) \rightarrow \sim(A \rightarrow \sim B)$, in [25, p. 396] Routley first presents McCall's [15] argument that makes use of the definition of compatibility, $(A \diamond B) \leftrightarrow$ def $\sim(A \rightarrow \sim B)$ and the assumption that $(A \rightarrow B) \rightarrow(A \diamond B)$ is valid, and then turns to a "slightly deeper argument" for the latter assumption, bringing negation as cancellation into play (notation adjusted):

\footnotetext{
${ }^{7}$ Routley [26, p. 82] explains that Addition, $A \rightarrow(A \vee B)$, is "generally rejected along with Simplification, since Contraposition and De Morgan principles are generally accepted." However, the generality of acceptance of Contraposition and the De Morgan principles may be questioned, as intuitionists do not accept all De Morgan laws, and, moreover, Contraposition, both in rule form, i.e., from $A \rightarrow B$ infer $\sim B \rightarrow \sim A$, and as an axiom, is rejected in David Nelson's constructive logics with strong negation and their substructural subsystems.
}

Australasian Journal of Logic (15:2) 2018 Article no. 3.11 
if $A$ is incompatible with $B$ then $A \wedge B$ says less than $A$ and than $B$ because $B$ has negated and so cancelled part of $A$; thus $A \wedge B$ does not imply $A$ and does not imply $B$, so $A$ does not imply $B$. In short the cancellation account of negation, in combination with a content account of implication, especially one which connects $A \rightarrow B$ with $(A \wedge B) \leftrightarrow A$, vindicates Boethius.

Given that the cancellation account of negation is afflicted with serious problems, we may stress that Boethius' thesis does not display conjunction and can be and has been motivated independently of the idea of negation as cancellation or deletion, according to which $(A \wedge \sim A)$ and $(\sim A \wedge A)$ are void of content. In the paraconsistent and constructive connexive logic $\mathbf{C}$ from [31], Boethius' thesis is arrived at by modifying the falsity conditions of constructive implication in David Nelson's constructive four-valued Logic N4 $[1,17,12]$. In classical and intuitionistic logic $\sim(A \rightarrow B)$ is logically equivalent to $A \wedge \sim B$, in $\mathbf{C}$, however, $\sim(A \rightarrow B)$ is logically equivalent to $A \rightarrow \sim B$ : a state in a model supports the falsity of $A \rightarrow B$ iff it supports the truth of $A \rightarrow \sim B$. In $\mathbf{C}$ the double negation laws, all De Morgan laws, the following versions of Aristotle's Theses and Boethius' Theses: ${ }^{8}$

$$
\begin{array}{ll}
\mathrm{AT} & \sim(\sim A \rightarrow A), \\
\mathrm{AT}^{\prime} & \sim(A \rightarrow \sim A), \\
\mathrm{BT} & (A \rightarrow B) \rightarrow \sim(A \rightarrow \sim B), \\
\mathrm{BT}^{\prime} & (A \rightarrow \sim B) \rightarrow \sim(A \rightarrow B)
\end{array}
$$

as well as the converses of $\mathrm{BT}$ and $\mathrm{BT}^{\prime}$ are valid.

\section{Digression}

Storrs McCall $\left[16\right.$, p. 446] doubts that the converse of $\mathrm{BT}^{\prime}, \sim(A \rightarrow B) \rightarrow(A \rightarrow \sim B)$, is a connexive thesis. Here is his argument.

Wansing's system cannot be regarded as a "basic" system of connexive logic. The difficulty lies in the converse of Boethius' thesis:

(4) $\sim(A \rightarrow B) \rightarrow(A \rightarrow \sim B)$.

(4) is a highly unintuitive implicational thesis, to which many counter-examples spring to mind. For example, it may be true that:

(5) It is not the case that if snow is white, grass is green,

but it is surely not the case that:

(6) If snow is white, then grass is not green.

If the price of having a "basic" system of connexive logic is accepting the implication of (6) by (5), then that price is simply too high to pay. If connexive implication is based in some way or other on a "connection" between the antecedent and consequent of any true implication, then this connection is palpably lacking in (6).

McCall argues that since there is no "connection" between the antecedent and consequent of (6), this sentence is not true for a connexive reading of the conditional occurring in it. Sentence (6) is an interpreted sentence: "snow" means snow, "grass" denotes grass, and since the colour of snow is independent of the colour of grass, one might think that the antecedent and consequent of (6) are not "connected". However, it makes a substantial difference whether one requires a connection between the antecedent and consequent of true implications or logically valid implications. Working with the latter requirement, as in [32], the

\footnotetext{
${ }^{8}$ Routley [26, p. 93] attests connexive logicians a tendency towards semantic holism: "Connectives interact with each other in a way that defeats natural separation of the role of connectives." In C, connectives play different roles depending on whether support of truth or support of falsity is concerned. These roles are clearly separated insofar as for every connective separate support of truth and support of falsity conditions are stated for formulas with that connective as the main logical symbol.
}

Australasian Journal of Logic (15:2) 2018 Article no. 3.11 
supposed lack of connection in (6) is irrelevant, so that even under a connexive reading of the conditional in that sentence, there are models that support the truth of "If snow is white, then grass is not green".

It has never been maintained that the strong negation, , of $\mathbf{C}$ captures every use of the word "not" in English, but it has been claimed that the strong negation in $\mathbf{C}$ expresses falsity. Let us therefore assume that the "not" in (5) expresses falsity. What are the falsity conditions of "If snow is white, grass is green"? If the falsity conditions familiar from classical or intuitionistic logic are assumed, instead of those of $\mathbf{C}$, then "If snow is white, grass is green" is false just in case snow is white and grass is not green. But this entails not only in classical logic, but also in intuitionistic logic as well as in $\mathbf{C}$, that if snow is white, then grass is not green. McCall's alleged counterexample vanishes.

End of Digression

The reason why the Routleys consider the failure of Simplification as characteristic of connexive logic is not only that they take both the failure of Simplification and Aristotle's and Boethius' theses to be justified by the idea of negation as cancellation. Routley [26, p. 82] writes that "[e]vidently, thesis 2 guarantees thesis 1", which may also be seen as indicating that for Routley, the validity of Boethius' and Aristotle's theses enjoys primacy over the failure of Simplification as a leading idea of connexive logic. As Routley points out, Boethius' thesis BT together with Simplification (and Modus Ponens) leads to negation inconsistency: from $(A \wedge \sim A) \rightarrow A$ and the following instance of BT; $((A \wedge \sim A) \rightarrow A) \rightarrow \sim((A \wedge \sim A) \rightarrow \sim A)$, one obtains $\sim((A \wedge \sim A) \rightarrow \sim A)$, which contradicts $(A \wedge \sim A) \rightarrow \sim A$. The connexive logic $\mathbf{C}$ is paraconsistent, and although it does validate BT and Simplification, the logic is not trivial. This shows that connexive logic can be freed from the idea of negation as cancellation by rejecting the highly unintuitive failure of Simplification. This liberation comes at the cost of negation inconsistency but not necessarily at the cost of triviality. ${ }^{9}$ Failure of Simplification is simply not a characteristic of connexive logic, and we thus agree with McCall [16, p. 445] when he writes that "the idée maitresse of connexive logic lies elsewhere."

However, McCall also considers the principles which he calls "Abelard's first principle" and "Aristotle's second thesis" as connexive principles:

- Abelard's First Principle: $\sim((A \rightarrow B) \wedge(A \rightarrow \sim B))$,

- Aristotle's Second Thesis: $\sim((A \rightarrow B) \wedge(\sim A \rightarrow B))$.

Routley [25, p. 394] refers to Abelard's First Principle as Strawson's principle (actually, he refers to it just as as "Strawson"), and presents an argument, ascribed to Aristotle and discussed by Jan Łukasiewicz [13], for that principle as follows (notation adjusted):

$$
\begin{aligned}
"((A \rightarrow B) \wedge(A \rightarrow \sim B)) & \rightarrow((A \rightarrow B) \wedge(B \rightarrow \sim A)) \\
& \rightarrow(A \rightarrow \sim A)
\end{aligned}
$$

Hence as $\sim(A \rightarrow \sim A), \sim((A \rightarrow B) \rightarrow(A \rightarrow \sim B))$ ". This derivation of Abelard's First Principle from one of Aristotle's theses makes a number of assumptions, such as the correctness of a rule of negation introduction that does not hold for strong negation in the connexive logic C. It is pointed out in [32] that Abelard's First Principle and Aristotle's Second Thesis are interderivable with BT and with BT' using intuitionistic principles only. Interesting as this may be, reading $\sim$ as intuitionistic negation and $\rightarrow$ as intuitionistic implication can hardly justify Aristotle's Second Thesis and Abelard's First Principle as connexive theses, as intuitionistic logic fails to validate BT, and BT'. Since Aristotle's Second Thesis and Abelard's First Principle both involve conjunction, it is tempting to obtain motivation for them from the idea of negation as cancellation and from the failure of Simplification as justified by the cancellation model of negation. Abelard's First Principle negates in particular the conjunction of instances of Simplification that are ruled out by negation as cancellation: $((B \wedge \sim B) \rightarrow B) \wedge((B \wedge \sim B) \rightarrow \sim B)$. If indeed Addition, $A \rightarrow(A \vee B)$ and $B \rightarrow(A \vee B)$, is rejected along with Simplification (see Footnote 7) because Contraposition and the De

\footnotetext{
${ }^{9}$ Note that this comment is not meant to say that in general non-trivial inconsistent systems should be preferred over logics failing Simplification, although failure of Simplification is indeed unintuitive.
}

Australasian Journal of Logic (15:2) 2018 Article no. 3.11 
Morgan laws are accepted, Aristotle's Second Thesis negates in particular the conjunction of instances of Addition that must be ruled out because of failure of Simplification: $(B \rightarrow(B \vee \sim B)) \wedge(\sim B \rightarrow(B \vee \sim B))$. It does not come as a surprise that in the system $\mathbf{C}$, in which Aristotle's and Boethius' Theses are not motivated by negation as cancellation, Aristotle's Second Thesis and Abelard's First Principle fail to be valid. In summary we may conclude that what is characteristic of connexive logic is the interplay between implication and negation displayed by $\mathrm{AT}, \mathrm{AT}^{\prime}, \mathrm{BT}$, and $\mathrm{BT}^{\prime}$.

\section{Ex CONTRAdictione NON SEQuitur CONTRAdictio}

The idea of inferential ineffectiveness of contradictions can be conceptually detached from the notion of negation as cancellation as well, if we focus on the inferential role of contradictions in the quote from $[27$, p. 205]:

$\sim A$ deletes, neutralizes, erases, cancels $A$ (and similarly, since the relation is symmetrical, $A$ erases $\sim A$ ), so that $\sim A$ together with $A$ leaves nothing, no content. The conjunction of $A$ and $\sim A$ says nothing, so nothing more specific follows. In particular, $A \wedge \sim A$ does not entail $A$ and does not entail $\sim A$.

Of course, the sentence " $\sim A$ together with $A$ leaves nothing, no content" seems to say that indeed nothing at all follows from a contradictory pair $A$ and $\sim A$, i.e., a contradiction cancels out anything.

However, the inferential ineffectiveness of contradictions is not necessarily bound to an account of negation as cancellation. According to Routley and Routley [27, p. 205] "[c]ontradictions may be allowed no inferential role [...], a total inferential role [...], or some limited inferential role [...]." And even though the idea that contradictions have no inferential role can be interpreted as being equivalent to an account of negation as cancellation, Routley himself, on the other hand, can be understood as endorsing the view that some contradictions or inconsistent premise sets may have a limited inferential role instead of none, if we read the quote from [26, p. 92] differently:

$\sim A$ deletes $A$ content, and $A$ and $\sim A$ together say nothing $A$-wise, i.e. $A \wedge \sim A$ has no $A$ content. But to say $A \wedge \sim A$ have no $A$ content is not to say it has no $B$ content [...].

Take, for example, the classically inconsistent set of formulas $\{A, \sim A, B\}$. Clearly, if we do not want inconsistent premise sets or premise sets containing contradictions to have any inferential power, then neither $A, \sim A, B \vDash A^{10}$ nor $A, \sim A, B \vDash \sim A$. However, to say that contradictions are inferentially ineffective is different from an account of negation as cancellation. If we accept the view that " $A$ and $\sim A$ together say nothing $A$-wise", one still might ask, "What about $B$ ?" Should $B$ be inferred from $\{A, \sim A, B\}$ ?

In this section, we will assume that contradictions are inferentially ineffective and thus are realizing ex contradictione non sequitur contradictio, without committing ourselves to an account of negation as cancellation. We will show that the negation of Priest's non-monotonic minimally inconsistent $L P$ $(L P m)$, cf. [24, p. 221], together with Grzegorz Malinowski's non-reflexive q-entailment, cf. [14], leads to a paraconsistent negation that satisfies Routley's principal idea of a negation so that " $A$ and $\sim A$ together say nothing $A$-wise".

The resulting logic, $q L P m$, will be non-monotonic and non-reflexive. As to non-monotonicity of the semantic consequence, Priest noted that "[t]his is as it should be with negation as cancellation, since it is part of the very conception that adding premises may, in fact, take away information", cf. [23, p. 143]. However, non-monotonicity of inference also already emerges if one just treats contradictions as inferentially ineffective.

As to non-reflexivity of the semantic consequence relation, if we do want that contradictions are inferentially ineffective, i.e., nothing contradictory follows from a contradiction, then we clearly do not want $A \wedge \sim A \vDash A \wedge \sim A$ to be valid. Contrary to Routley's clear rejection of Simplification, " $A \wedge \sim A$ does

\footnotetext{
${ }^{10}$ We omit brackets for sets of formulas on the left side of $\vDash$.
} 
not entail $A$ and does not entail $\sim A$," he was less clear about non-reflexivity: "Contradictions may be allowed no inferential role (they imply nothing, except perhaps themselves)". A moderate reading of this quote would allow contradictions to imply at least themselves. Our approach is strict in the sense that contradictions fail to imply not only their conjuncts but also themselves, but moderate enough such that something can follow from a contradiction, namely formulas where the valuation is not affected by the contradiction.

There are at least three other systems, besides the one presented in this article, which also endorse some kind of inferential ineffectiveness of contradictions. As early as in 1976, Frederick Johnson [11] described a system he calls $R C$, with the aim to provide a three-valued semantics for a relevance logic. There he combined Bochvar's logic $B_{3}$, cf. [4], with a non-standard consequence relation and obtained a system that is non-monotonic, non-reflexive, without Simplification, and that countenances an account of negation, where nothing at all follows from a contradiction. In [23] Priest described a logical system with an account of negation as cancellation. Although not promoting the cancellation account, Priest showed that some weak connexive principles emerge as a consequence from such an approach. It is interesting to note that Thomas Macaulay Ferguson showed in [8] that Johnson's system is just the intersection of Bochvar's $B_{3}$ and Priest's approach. Another recent system is described by Adam Přenosil in [20]. There, Přenosil develops a non-monotonic version of $L P$, different from the one described below, to generate a moderate account of negation as cancellation in order to deal with contradictory information in systems of belief revision.

It is generally interesting to mention that in the area of computer science, and non-monotonic reasoning in particular, the idea that contradictions should not have any inferential power is not new and has been suggested with no reference to the cancellation model of negation. Donald Perlis [19], for instance, explains that "when a (direct) contradiction is found in our reasoning, we tend to notice that fact and take corrective action, such as temporarily suspending belief in one or both conflicting beliefs." In Gerd Wagner's article Ex contradictione nihil sequitur [30] we find the following passage:

From an information processing point of view, however, it seems to be more natural to discard contradictory information as neither true nor false, so it cannot be used in the derivation of further information.

But also if contradictory information is seen as receiving both support of truth and support of falsity, it may be classified as unreliable (or in need of clarification or correction) and therefore as being inferentially ineffective. The approach presented here follows a similar path by developing a system where contradictory formulas have no influence on valid inferences, i.e., the valuation of contradictory formulas does not affect the semantical value of tautologies or the value of conclusions of valid inferences.

In the following, we will first recapitulate the definitions of minimal $L P$ and q-entailment and then demonstrate how the combination of both leads to a different account of inferentially ineffective contradictions. Furthermore, it was emphasized in [23] and [25, 27] that the cancellation account of negation leads towards connexive principles. It will be shown that the system $q L P m$ satisfies some weaker versions of those principles, while rejecting others. This is, however, not due to an account of negation as cancellation but to the non-standard definition of the consequence relation.

4.1. qLPm. The language $\mathcal{L}$ of $L P$, cf. [21], consists of the set $\{\neg, \wedge, \vee\}$ of primitive connectives, where $\rightarrow$ is defined as material implication, and a countable set Prop of propositional atoms $p, q$, etc. Furthermore, we denote by Form the set of formulas of our language constructed as usual. We denote formulas by $H$, $H_{1}, H_{2}$, etc. and sets of formulas by $\Sigma, \Gamma$, etc.

Definition 1. A matrix for $L P$ is a tuple $M=\langle\mathcal{V}, \mathcal{D}, \mathcal{O}\rangle$, where:

(a) $\mathcal{V}=\left\{0, \frac{1}{2}, 1\right\}$

(b) $\mathcal{D}=\left\{1, \frac{1}{2}\right\}$,

Australasian Journal of Logic (15:2) 2018 Article no. 3.11 
(c) For $\neg, \wedge, \vee$ of our language, $\mathcal{O}$ includes a corresponding $n$-ary function from $\mathcal{V}^{n}$ to $\mathcal{V}$ as follows:

$$
\tilde{\neg}(x)=1-x \quad \tilde{\wedge}(x, y)=\min (x, y) \quad \tilde{\mathrm{v}}(x, y)=\max (x, y)
$$

A valuation in a matrix $M$ is a function $v:$ Form $\rightarrow \mathcal{V}$ that satisfies the following condition for every $n$-ary connective $*$ of $\mathcal{L}$ and $H_{1}, \ldots, H_{n} \in$ Form:

$$
v\left(*\left(H_{1}, \ldots, H_{n}\right)\right)=\tilde{*}\left(v\left(H_{1}\right), \ldots, v\left(H_{n}\right)\right)
$$

A valuation $v$ is called an $L P$ model of $H(\Sigma)$ iff $v(H) \in \mathcal{D}(v(G) \in \mathcal{D}$ for all $G \in \Sigma)$.

Definition 2. A formula $H$ is an $L P$ consequence of $\Sigma\left(\Sigma \vDash_{L P} H\right)$ iff for all valuations $v$, if $v$ is a model of $\Sigma$, then $v$ is a model of $H$. In particular, $H$ is an $L P$ tautology iff $v$ is a model of $H$ for all valuations $v$.

It is well known that $L P$ is paraconsistent and lacks Modus Ponens and the Disjunctive Syllogism, while all classical tautologies are tautologies of $L P$ as well, and vice versa. In order to overcome the lack of useful deduction rules, which could be seen as a shortcoming of $L P$, Priest developed a non-monotonic version of $L P$, namely minimally inconsistent $L P$, cf. [22] and [24, Chapter 16].

Definition 3. Let $\Sigma$ be a set of sentences and $v_{1}, v_{2}$ LP models of $\Sigma$. We define an ordering, <, on the set of all models of $\Sigma$ in the following way: $v_{1}<v_{2}$ iff

(1) if $v_{1}(p)=\frac{1}{2}$ then $v_{2}(p)=\frac{1}{2}$ for all propositional atoms contained in $\Sigma$,

(2) there is at least one propositional atom $q$ occuring in $\Sigma$, such that $v_{2}(q)=\frac{1}{2}$, but $v_{1}(q) \neq \frac{1}{2}$.

A model $v_{1}$ is called minimal (or LPm model) iff there is no model $v_{2}$ such that $v_{2}<v_{1}$.

Definition 4. A formula $H$ is an $L P m$ consequence of $\Sigma\left(\Sigma \vDash_{L P m} H\right)$ iff all $L P m$ models $v$ of $\Sigma$ are models of $H$.

$L P m$ is well-defined at least in the propositional case, i.e., for every class of models of some set of formulas $\Sigma$ there are minimal models of $\Sigma$. However, the case of the first-order version of $L P m$ is more difficult, cf. [6] and [7] for a discussion on first-order LPm. Nevertheless, in this section we will restrict ourselves to the propositional case. It is possible to develop a first-order version of the non-monotonic logic $q L P m$, introduced below, but we leave this topic for another time.

With the definition of LPm at hand, we can now distinguish between defective and normal propositional atoms. We will use this distinction to clarify what is effective in valid inferences and what is not.

Definition 5. Let $\Sigma$ be a set of sentences and $V$ be the class of all $L P m$ models of $\Sigma$. A propositional atom $p$ is called defective with respect to $\Sigma$, iff $v(p)=\frac{1}{2}$ for all $v \in V$. A propositional atom $p$ is normal with respect to $\Sigma$ iff it is not defective.

The definition of defective propositional atoms alone does not lead to an account of contradictions being inferentially ineffective, since in $L P m$ we have $p, \neg p \vDash_{L P m} p$. In order to make defective propositions inferentially ineffective, we will make use of so-called q-consequences ("quasi-consequences"). Quasiconsequence relations were introduced by Malinowski in [14], to show that by generalizing Tarski's definition of consequence relations, it is indeed possible to construct genuine three-valued logics, the algebraic semantics of which cannot be reduced to a bivalent one. This result challenges Suszko's thesis, according to which there are only two logical values, cf. $[28,3]$. Single-conclusion q-entailment, $\vDash_{q}$, is defined by requiring that if every premise is not anti-designated, then the conclusion is designated. Whereas in matrices for $L P$ the set of designated values $\mathcal{D}=\left\{1, \frac{1}{2}\right\}$, for $q L P m$ consequences, the set of designated values $\mathcal{D}=\{1\}$, and the set of anti-designated values is $\{0\}$, so that $\left\{1, \frac{1}{2}\right\}$ is the set of not anti-designated values.

Australasian Journal of Logic (15:2) 2018 Article no. 3.11 
Definition 6. A formula $H$ is a $q L P m$ consequence of $\Sigma\left(\Sigma \vDash_{q L P m} H\right)$ iff for all minimal models $v$ of $\Sigma$, if $v(G) \in\left\{1, \frac{1}{2}\right\}$ for all $B \in \Sigma$ then $v(H)=1$. In particular, $H$ is a $q L P m$ tautology $\left(\vDash_{q L P m} H\right)$ iff for all minimal models $v, v(H)=1$.

In the next section, we will list some important properties of $q L P m$.

4.2. Properties of $\mathbf{q} \mathbf{L P m}$. The first lemma shows that nothing classically contradictory follows from a classically inconsistent premise set.

Lemma 7. Let $\Sigma$ be a set of formulas and let $p$ be defective with respect to $\Sigma$, then $\Sigma \sharp_{q L P m} p$.

Proof. Let $\Sigma$ be a set of formulas and let $p$ be defective with respect to $\Sigma$, i.e., $v(p)=\frac{1}{2}$ for all $L P m$ models $v$ of $\Sigma$, hence for no such $v, v(p)=1$ and therefore $\Sigma \#_{q L P m} p$.

The following examples show some valid and some invalid inferences of $q L P m$.

Example 8. Let $q, p$ be propositional atoms, then

- $p \wedge \neg p \#_{q L P m} p, p \wedge \neg p \#_{q L P m} \neg p$ and $p \wedge \neg p \#_{q L P m} p \wedge \neg p$,

- $\vDash_{q L P m} p \vee \neg p$, but $p \wedge \neg p \#_{q L P m} p \vee \neg p$, as well as $p \wedge \neg p \#_{q L P m} p \rightarrow p$,

- $p \wedge \neg p \wedge q \vDash_{q L P m} q$ and $p \wedge \neg p \wedge q \vDash_{q L P m} q \vee p$,

- $p \wedge \neg p \vDash_{q L P m} q \rightarrow(p \rightarrow q)$ but $p \wedge \neg p \vDash_{q L P m} p \rightarrow(q \rightarrow p)$.

Now, even though we made use of a combination of $L P m$ and q-consequences, the consequences of $q L P m$ are those of classical logic, in consistent contexts.

Lemma 9. If $\Sigma$ is classically consistent, then $\Sigma \vDash_{q L P m} H$ iff $\Sigma \vDash_{C L} H$, where $\vDash_{C L}$ is the consequence relation of classical propositional logic. In particular, $\vDash_{q L P m} H$ iff $\vDash_{C L} H$, i.e., the tautologies of classical propositional logic are the tautologies of $q L P m$.

Proof. This lemma follows immediately in light of the results for LPm, cf. [24, Chapter 16], [6] and [7].

The previous lemma claims that for $q L P m$ we have a recapture of classical consequences in consistent contexts, in particular, the set of tautologies of $q L P m$ is the same as in classical logic. However, as Example 8 shows, we lose some tautologies as consequences of classically inconsistent premise sets. Example 8 also shows another interesting property. Even though Lemma 7 guarantees that a defective propositional atom $p$ cannot be inferred directly, it still might be the case that $p$ occurs in some subformula of the conclusion of a valid inference. However, if $p$ is defective and occurs in some conclusion $H$, then $p$ has no inferential power, i.e., if $\Sigma \cup\{H\}$ is a set of formulas, $\Sigma \vDash_{q L P m} H$ and $p$ is defective with respect to $\Sigma$, then, if $p$ occurs in $H$, the value of $p$ does not affect the value of $H$.

4.3. qLPm and Connexive Principles. Routley and Routley [27, p. 205] and Priest [23] discuss the relation between negation as cancellation and connexive logic. Even though we are, as pointed out earlier, skeptical about their motivation, it is interesting to note that $q L P m$ shares some weak connection with connexive logic on the level of the consequence relation.

Note that there is some clutter when it comes to naming certain connexive principles (and to classifying certain principles as connexive). In the following, we will use Priest's notation from [23, p. 143]:

(Ar1) it is not the case that: $\neg H \vDash H$,

(Ar2) it is not the case that: $H \vDash \neg H$,

(Ab1) it is not the case that: $H \vDash G$ and $\neg H \vDash G$,

(Ab2) it is not the case that: $H \vDash G$ and $H \vDash \neg G$,

(Bo1) if $H \vDash G$, then it is not the case that: $\neg H \vDash G$,

(Bo2) if $H \vDash G$, then it is not the case that: $H \vDash \neg G$.

Lemma 10. (Ar1), (Ar2), (Ab2) and (Bo2) hold for $q L P m$, while (Ab1) and (Bo1) do not. 
Proof. To show that (Ab1) and (Bo1) do not hold for $q L P m$, just take as $G$ any tautology, sharing no propositional atoms with $H$.

As for (Ar1), assume for contradiction that $\neg H \vDash H$ for some $H$. Then, if $v(\neg H) \in\left\{1, \frac{1}{2}\right\}$, then $v(H)=1$. But this is impossible, since, if $v(\neg H) \in\left\{1, \frac{1}{2}\right\}$, then $v(H) \in\left\{0, \frac{1}{2}\right\}$. Hence, it is not the case that $H$ follows from $\neg H$.

The proofs for (Ar2), (Ab2) and (Bo2) make use of similar arguments and are therefore left to the reader.

Of course, the principles (AR1) - (Bo2) can be seen as not being proper connexive principles since they involve two kinds of negations. One is in the meta-language and the other is in the object language, while in proper connexive theses there is only one kind of negation in the object language. ${ }^{11}$

Connexive logic is always thought of as being orthogonal to classical logic with respect to the set of theorems. However, as was shown, $q L P m$, which has the same set of tautologies as classical logic, shares some principles with connexive logic on the level of the consequence relation. This is not surprising in light of the observations made by Ferguson in [8], who showed that even classical logic shares some weak connexive principles at the level of consequence, when only contingent formulas are involved. ${ }^{12}$ It should be clear at this point that we cannot find convincing arguments in favor of negation as cancellation as a basis for connexive logic. The weak connexive principles that hold in the systems described in [8], [11], [20], [23] and in this article are rather a byproduct of non-standard definitions of the consequence relations.

\section{Conclusion}

In this article, we reflected on Richard and Valery Routley's discussion of the notion of negation as cancellation. We first clarified that the concept of negation as cancellation is highly problematic. Secondly, because of the first point, we think that negation as cancellation should not be taken as a conceptional basis of anything and particularly not as a basis of connexive logic. Furthermore, with the non-monotonic logic $q L P m$, we developed a system in which contradictions are inferentially ineffective in a certain sense and thereby we detached the concept of negation as cancellation from the idea of assigning no inferential role to contradictions.

\section{ACKNowledgements}

We are grateful to two anonymous referees for their very detailed, critical, and helpful comments.

\section{REFERENCES}

[1] Ahmad Almukdad and David Nelson. Constructible falsity and inexact predicates. Journal of Symbolic Logic, 49(1):231233, 1984.

[2] Franz Berto. Aboutness in imagination. Philosophical Studies, 2017, https://doi.org/10.1007/s11098-017-0937-y.

[3] Carolina Blasio, João Marcos, and Heinrich Wansing. An inferentially many-valued two-dimensional notion of entailment. Bulletin of the Section of Logic, 46:233-262, 2017.

[4] D.A. Bochvar and Merrie Bergmann. On a three-valued logical calculus and its application to the analysis of the paradoxes of the classical extended functional calculus. History and Philosophy of Logic, 2(1-2):87-112, 1981.

[5] Pablo Cobreros, Paul Egré, David Ripley, and Robert van Rooij. Tolerant, classical, strict. Journal of Philosophical Logic, 41(2):347-385, 2012.

[6] Marcel Crabbé. Reassurance for the logic of paradox. Review of Symbolic Logic, 4(3):479-485, 2011.

[7] Marcel Crabbé. Reassurance via translation. Logique Et Analyse, 55(218):281-293, 2012.

[8] Thomas Macaulay Ferguson. Logics of nonsense and Parry systems. Journal of Philosophical Logic, 44(1):65-80, Feb 2015.

11 [8], [23] seem to take the weak connexive principles at the consequence level as proper connexive principles.

12 Please note that similar observations can be made for $L P m$. 
[9] Thomas Macaulay Ferguson. Meaning and Proscription in Formal Logic. Variations on the Propositional Logic of William T. Parry. Springer, Dordrecht, 2017.

[10] Patrick Grimm. What is a contradiction? In Graham Priest, Jc Beall, and Bradley Armour-Garb, editors, The Law of Non-Contradiction: New Philosophcial Essays, pages 49-72. Oxford University Press, 2005.

[11] Fred Johnson. A three-valued interpretation for a relevance logic. The Relevance Logic Newsletter, 1(3):123-128, 1976.

[12] Norihiro Kamide and Heinrich Wansing. Proof Theory of N4-related Paraconsistent Logics. College Publications, London, 2015.

[13] Jan Łukasiewicz. Aristotle's Syllogistic, Second enlarged edition. Clarendon Press, Oxford, 1957.

[14] Grzegorz Malinowski. Inferential many-valuedness. In Jan Wolenski, editor, Philosophical Logic in Poland, pages 75-84. Kluwer Academic Publishers, Dordrecht, 1994.

[15] Storrs McCall. Connexive implication. Journal of Symbolic Logic, 31:415-433, 1966.

[16] Storrs McCall. A history of connexivity. In Dov Gabbay et al., editor, A History of Logic, volume 11, pages 415-449. Elsevier, 2012.

[17] Sergei P. Odintsov. Constructive Negations and Paraconsistency. Springer-Verlag, Dordrecht, 2008.

[18] Hitoshi Omori and Heinrich Wansing. 40 years of FDE: An introductory overview. Studia Logica, 105(6):1021-1049, 2017.

[19] Donald Perlis. Truth and meaning. Artificial Intelligence, 39(2):245 - 250, 1989.

[20] Adam Přenosil. Contradictory information as a basis for rational belief. In Alexandru Baltag, Jeremy Seligman, and Tomoyuki Yamada, editors, Logic, Rationality, and Interaction, pages 151-165, Berlin, Heidelberg, 2017. Springer Berlin Heidelberg.

[21] Graham Priest. The logic of paradox. Journal of Philosophical logic, 8(1):219-241, 1979.

[22] Graham Priest. Minimally inconsistent LP. Studia Logica, 50(2):321-331, 1991.

[23] Graham Priest. Negation as cancellation, and connexive logic. Topoi, 18(2):141-148, 1999.

[24] Graham Priest. In Contradiction: A Study of the Transconsistent. Oxford University Press, 2006.

[25] Richard Routley. Semantics for connexive logics. I. Studia Logica, 37(4):339-412, 1978.

[26] Richard Routley, Robert K. Meyer, Val Plumwood, and Ross T. Brady. Relevant Logics and their Rivals, Vol. 1. Ridgeview Publishing Company, Atascadero, 1982.

[27] Richard Routley and Valerie Routley. Negation and contradiction. Revista Colombiana de Matemáticas, 19(1-2):201-230, 1985.

[28] Roman Suszko. Remarks on Lukasiewicz's three-valued logic. Bulletin of the Section of Logic, 4:87-90, 1975.

[29] Richard Sylvan. Relevant containment logics and certain frame problems of AI. Logique et Analyse, 31(121-122):11-25, 1988.

[30] Gerd Wagner. Ex contradictione nihil sequitur. In Proceedings of the 12th International Joint Conference on Artificial Intelligence - Volume 1, IJCAI'91, pages 538-543, San Francisco, CA, USA, 1991. Morgan Kaufmann Publishers Inc.

[31] Heinrich Wansing. Connexive Modal Logic. In Renate Schmidt et al., editor, Advances in Modal Logic, volume 5, pages 367-383. King's Colledge Publications, London, 2005.

[32] Heinrich Wansing. Connexive logic. In Edward N. Zalta, editor, The Stanford Encyclopedia of Philosophy. Metaphysics Research Lab, Stanford University, spring 2016 edition, 2016.

[33] Stephen Yablo. Aboutness. Princeton University Press, Princeton, 2014.

Department of Philosophy II, Ruhr-Universität Bochum, Germany

E-mail address: heinrich.wansing@rub.de

Department of Philosophy II, Ruhr-Universität Bochum, Germany

E-mail address: daniel.skurt@rub.de

Australasian Journal of Logic (15:2) 2018 Article no. 3.11 